Becerra Traver, M.T. (2017). La habilidad de hablar en público. Una experiencia formativa con estudiantes universitarios. Revista Electrónica Interuniversitaria de Formación del Profesorado, 20 (3), 117-129. DOI: http://dx.doi.org/10.6018/reifop.20.3.270631

\title{
La habilidad de hablar en público. Una experiencia formativa con estudiantes universitarios
}

\author{
Maria Teresa Becerra Traver \\ Facultad de Educación. Universidad de Extremadura
}

\section{Resumen}

La consolidación del Espacio Europeo de Educación Superior (EEES) en el ámbito universitario ha supuesto notables cambios en la formación del alumnado. En esta experiencia formativa se ha intentado dotar al alumnado universitario del Grado de Educación Primaria de la Facultad de Educación de la Universidad de Extremadura de los recursos necesarios que le permitan desarrollar la competencia transversal de hablar en público. Los resultados extraídos nos llevan a considerar la posibilidad de ofertar un taller a los estudiantes con objeto de contribuir al desarrollo de la competencia transversal de hablar en público, y por tanto, a enriquecer sus potenciales como docentes.

\section{Palabras clave}

Interacción; hablar en público; competencia transversal; universidad.

\section{Public speaking skill. A teaching experience with universitary students}

\begin{abstract}
The consolidation of the European Higher Education Area (EHEA) in universities has led to significant changes in student training. In this teaching experience we intend to provide resources that enable university students from Teacher Degree in Education Faculty of University of Extremadura to develop the cross curricular competence of public speaking. The results lead us to discuss the possibility of offering a workshop to students in order to contribute to the development of cross curricular competence of public speaking, and to enrich their potentialities as teachers.
\end{abstract}

\section{Key words}

Interaction; public Speaking; cross curricular competence; university. 


\section{Introducción}

En España, la consolidación del Espacio Europeo de Educación Superior (EEES) en el ámbito universitario ha supuesto notables cambios en la formación del alumnado. Estos cambios promueven la autonomía, el desarrollo de competencias y una formación general que parte del desarrollo académico, personal y profesional del estudiante (Castaño, Blanco \& Asensio, 2012). En concreto, hay que destacar la implantación de nuevos sistemas de evaluación, como por ejemplo el Trabajo Fin de Grado. Se trata de una asignatura que conlleva la elaboración de un trabajo escrito, dirigido por un profesor/a y presentado ante un Tribunal con el objeto de que el estudiante demuestre la puesta en práctica de las competencias asociadas a la Titulación y sirva para desarrollar una actividad autónoma, responsable y comprehensiva.

La realización de este Trabajo Fin de Grado (en adelante TFG) engloba una serie de competencias generales, específicas y transversales que el alumnado contribuirá a desarrollar y superar. Quizás, la mayor dificultad o, se podría decir, miedo que encuentra el alumnado en el desarrollo del TFG es la competencia transversal descrita en los correspondientes programas de esta asignatura referida a Presentar públicamente ideas, problemas y soluciones, de una manera lógica, estructurada, tanto oralmente como por escrito.

Esta competencia transversal de hablar en público no sólo se desarrolla en este momento clave de los estudios universitarios del alumnado sino que se puede presentar en algún momento de su vida. Ya sea para presentar un trabajo en clase ante el profesor y los compañeros, dar una opinión en una reunión de empresa, realizar una ponencia en un congreso científico... No obstante, aunque a muchas personas les gustaría dar su opinión o expresarse en público, su falta de habilidades o su miedo les cohíbe a la hora de hacerlo (Bados, 2005).

El filósofo José Antonio Marina (2006) en su libro Anatomía del miedo considera el miedo como una ansiedad provocada ante la anticipación de un peligro. Por su parte, ya en 1977 Wallace, Wallechinsky y Wallace en su primer libro publicado The Book of Lists recopila una estadística sobre los miedos de las personas. Esta estadística identifica en primer lugar el miedo a hablar en público, dejando por debajo de este, por ejemplo, el miedo a la enfermedad o el miedo a la muerte.

Diversos estudios corroboran que un 20-30\% de los estudiantes universitarios (Bados, 1992; D’El Rey \& Pacini, 2005; Lane \& Borkovec, 1984; Lang, Levin, Miller \& Kozak, 1983) informan tener un gran miedo a hablar en público. Intervenir o hablar en público es considerado por adultos y adolescentes como una de las situaciones más difíciles (Argyle, 1981; Harris \& Brown, 1982; Stein, Walker \& Forde, 1994).

Bang-Rouhet (2009) parte de la idea de que escuchando a un orador se puede descubrir su historia personal a través de los gestos, la mirada, el volumen de voz y las entonaciones. La experiencia como docente y miembro de tribunales de los TFG, me han permitido, al igual que a otros compañeros, evidenciar las diversas reacciones que presenta el alumnado cuando se enfrenta a la exposición pública de un trabajo que tiene la finalidad de recibir una calificación. Se pueden presenciar reacciones como evitar el contacto visual, temblores en manos y piernas, sudoración de las palmas de las manos, falta de aire, rostro sonrojado, pérdida de la concentración, voz temblorosa o sequedad en la boca. Estos síntomas suelen ser consecuencia del miedo que les puede causar ser evaluados negativamente, no ser escuchados o valorados, quedarse en blanco y/o a la vergüenza que se pueda pasar frente a un posible fracaso. Por esto, el miedo a hablar en público se entiende como un déficit de habilidades para hablar en público (Marshall, 1981). Puchol (2008) señala que el éxito o 
fracaso para hablar en público depende fundamentalmente de la autoconfianza que tenga uno mismo, de la habilidad para comunicarse oral y gestualmente y de la confianza y simpatía que se transmita a la audiencia.

Como se ha visto, hoy en día los estudiantes universitarios se ven a menudo en la necesidad de exponer trabajos en público, ante sus profesores y compañeros. Se trata de una situación, relativamente nueva para algunos universitarios, en la que se ven expuestos a una valoración que generalmente recibe una calificación. Es esperable, por tanto, que aparezca un cierto nivel de miedo en cualquier alumno que se expone a esta situación. En algunos casos dicho nivel puede resultar excesivo para la persona, perjudicando notablemente su capacidad para hablar en público.

Ante esto, la Universidad debe brindar a los jóvenes las herramientas que les permitan interactuar positivamente con su entorno e insertarse de manera activa en la vida social, laboral y cultural. Hay que introducir nuevas metodologías que ayuden a contribuir al desarrollo de la competencia transversal de hablar en público.

La habilidad de hablar en público es algo que se puede aprender y mejorar con una formación y no sólo con la experiencia (Fernández, González, López \& Manso, 2010). En un estudio desarrollado por Olivares y García-López (2002) mostraron cómo los sujetos (estudiantes universitarias) que participaron en un tratamiento que integraba un entrenamiento en hablar en público, exposición, entrenamiento en autoinstrucciones y en respiración redujeron sus respuestas de ansiedad social. Por ello, es necesario dar respuesta a esta necesidad a partir del desarrollo en las aulas universitarias de experiencias educativas en las que el alumnado obtenga prácticas exitosas.

El estudio descriptivo que se expone a continuación presenta la experiencia formativa llevada a cabo con estudiantes universitarios en relación con la habilidad de hablar en público. El objetivo principal de esta experiencia era dotar al alumnado de la Facultad de Educación de la Universidad de Extremadura de los recursos necesarios que le permitiesen desarrollar la habilidad de hablar en público, y por consiguiente, que su nivel de miedo descendiese cuando tuviesen que hablar en público. Para ello, se impartió un taller compuesto de varias sesiones donde se trabajaron todos los aspectos de una presentación, desde la planificación teniendo en cuenta el propósito y la audiencia, pasando por el diseño visual de las diapositivas con Software de presentaciones (Software libre o Software de autor), hasta cómo prepararse, la presentación en sí, y cómo obtener retroalimentación de cómo se ha hecho. Más específicamente, se pretendía identificar los puntos fuertes y débiles del taller impartido, así como la valoración y repercusión de dicha experiencia formativa en los estudiantes universitarios.

\section{Metodología}

La experiencia formativa, realizada con alumnos de primero de Grado de Educación Primaria de la Facultad de Educación de la Universidad de Extremadura, se fundamentó en un diseño metodológico de tipo descriptivo en el que se establecen tres fases.

En la primera fase se impartió un taller, denominado Hablar en público, compuesto de varias sesiones donde se trabajaron todos los aspectos de una presentación, desde la planificación teniendo en cuenta el propósito y la audiencia, pasando por el diseño visual de las diapositivas con Software de presentaciones (Software libre o Software de autor), hasta cómo prepararse, la presentación en sí, y cómo obtener retroalimentación de cómo se ha hecho. En este taller participaron 141 estudiantes que asistían a la asignatura Recursos Tecnológicos Didácticos y de Investigación. 
En la segunda fase, una vez finalizado el taller, los alumnos asistentes expusieron un trabajo donde se evaluaban todos los aspectos desarrollados durante el taller. La evaluación de este trabajo se tendría en cuenta para la asignatura con la cual estaba relacionado el taller, Recursos Tecnológicos Didácticos y de Investigación. Los alumnos participantes hicieron una exposición, en grupos de cuatro a seis miembros, apoyándose en un software de presentaciones, donde presentaban un trabajo en el que se evaluaban los aspectos desarrollados en el taller, tales como:

- Discurso

- Estructura

- Exposición

- Diseño visual

- Control del tiempo

- Adecuación a la audiencia

- Comunicación verbal, no verbal...

Después de la exposición, cada alumno, a través de un cuestionario de satisfacción (véase anexo), valoró la formación recibida en dicho taller. Dicho cuestionario fue sometido a la valoración de expertos, mediante grupos de discusión. Se trata de un cuestionario autocumplimentado formado por 14 ítems que estaban agrupados en torno a tres secciones: (1) objetivos y contenidos trabajados en las sesiones; (2) metodología empleada y; (3) utilidad, valoración global del taller. Cada uno de los ítems del cuestionario tenía opciones de respuesta de una escala tipo Likert (1932) con cinco niveles de acuerdo o desacuerdo, siendo $1=$ Totalmente en desacuerdo, $2=$ En desacuerdo, $3=\mathrm{Ni}$ de acuerdo ni en desacuerdo, $4=$ De acuerdo y $5=$ Totalmente de acuerdo. Junto a estos ítems se añadieron una serie de preguntas de las cuales obtuvimos información sociodemográfica sobre los participantes (edad, sexo, curso...). Estos ítems genéricos se encuadran en una sección denominada Información personal.

La sección Objetivos y contenidos nos permite obtener información sobre si el taller impartido ha logrado las expectativas fijadas. Consta de los siguientes cuatro ítems: 1) En las sesiones realizadas se ha alcanzado el objetivo previsto: Mejorar la competencia transversal de hablar en público; 2) Los contenidos se han tratado de acuerdo al objetivo propuesto; 3) La duración del taller ha sido adecuada al objetivo propuesto; 4) La duración del taller ha sido adecuada a los contenidos desarrollados.

La sección Metodología ofrece información para comprobar si las estrategias didácticas y la metodología empleada son las adecuadas o, por el contrario, hay que introducir mejoras en próximos talleres. También está formada por cuatro ítems: 1) La metodología desarrollada se ha adecuado al objetivo propuesto; 2) La metodología desarrollada se ha adecuado a los contenidos expuestos; 3) El material didáctico utilizado ha sido adecuado para alcanzar el objetivo del taller; 4) La metodología ha permitido la participación activa de los asistentes.

La sección, Utilidad y valoración global, nos facilita información sobre la calidad del taller y de la repercusión en los participantes en la experiencia. Esta sección está compuesta por los siguientes seis ítems: 1) El taller recibido es importante para mi trabajo como docente; 2) Como alumno, me ha venido bien recibir este taller; 3) Recomendaría este taller a mis amigos; 4) Recomendaría este taller a mis compañeros de clase; 5) Creo que la exposición de mi trabajo ha resultado mejor al haber recibido el taller de hablar en público; 6) El taller merece una valoración global positiva. 
En la tercera fase, la información recogida de los cuestionarios fue analizada con el objetivo de identificar los puntos fuertes y débiles del taller impartido, así como la necesidad de hacer extensivo este tipo de taller al resto de estudiantes, en general, y a personas interesadas en desarrollar la habilidad de hablar en público, en particular. Para proceder con el análisis de los datos de este cuestionario se consideró en primer lugar cada uno de los apartados en los cuales se enmarcaban las preguntas: (1) objetivos y contenidos; (2) metodología y; (3) utilidad, valoración global.

En segundo lugar, se analizaron cada uno de los ítems de la encuesta. Esto nos llevó a observar la frecuencia con que aparecía cada respuesta en cada pregunta y, por lo tanto, a codificar la información y a agrupar los datos cuantitativos en frecuencias y porcentajes. No sólo se llevó a cabo el análisis teniendo en cuenta el conjunto de participantes sino que también se analizaron los datos por grupo de sexo (hombre y mujer).

Finalmente, se realizaron tablas para sintetizar la información, así como representaciones gráficas de los datos. A partir de aquí se pudo realizar una interpretación descriptiva de la información dentro del contexto en el que fue recogida y por tanto, extraer unas conclusiones.

\section{Resultados}

El procedimiento anteriormente descrito dio lugar a una amplia base de datos cuantitativos, cuyos resultados se muestran y explican detalladamente de acuerdo con las secciones que componen el cuestionario de satisfacción.

\section{Información personal}

En esta sección, como se ha comentado, se contemplan aquellas preguntas referidas a edad, sexo, curso... En concreto, en esta experiencia participaron un total de 141 alumnos (52 hombres y 89 mujeres) procedentes de la Facultad de Educación de la Universidad de Extremadura del Grado de Educación Primaria, con edades comprendidas entre los 18 y 38 años (véase tabla 1), siendo de los 18 a los 20 años el rango de edad más numeroso.

\section{Tabla 1.}

Edad de los alumnos participantes.

\begin{tabular}{|c|c|}
\hline Edad & Número de alumnos \\
\hline 18 & 36 \\
\hline 19 & 52 \\
\hline 20 & 30 \\
\hline 21 & 12 \\
\hline 22 & 2 \\
\hline 23 & 4 \\
\hline 24 & 3 \\
\hline 29 & 1 \\
\hline 38 & 1 \\
\hline
\end{tabular}

Fuente: Elaboración propia. 


\section{Objetivos y contenidos}

Como se puede apreciar en la tabla 2 y gráfico 1, el 62,41\% está de acuerdo en que el taller ofertado les ha permitido mejorar la competencia transversal de hablar en público. El 51,77\% considera que los contenidos se han tratado de acuerdo al objetivo propuesto. El 38,30\% está totalmente de acuerdo con la duración del taller para mejorar la competencia transversal de hablar en público. Un $45,39 \%$ si están de acuerdo y coinciden en que la duración del taller ha sido adecuada a los contenidos que se han desarrollado.

Tabla 2.

Sección Objetivos y contenidos.

\begin{tabular}{|l|c|c|c|c|c|}
\hline \multirow{2}{*}{ Ítem } & \multicolumn{5}{|c|}{ Nivel de acuerdo o desacuerdo } \\
\cline { 2 - 6 } & $\mathbf{1}$ & $\mathbf{2}$ & $\mathbf{3}$ & $\mathbf{4}$ & $\mathbf{5}$ \\
\hline $\begin{array}{l}\text { 2.1. En las sesiones realizadas se ha } \\
\text { alcanzado el objetivo previsto: Mejorar }\end{array}$ & $0,71 \%$ & $1,42 \%$ & $15,60 \%$ & $62,41 \%$ & $19,86 \%$ \\
$\begin{array}{l}\text { la competencia transversal de hablar } \\
\text { en público. }\end{array}$ & $0,00 \%$ & $1,42 \%$ & $7,09 \%$ & $51,77 \%$ & $39,72 \%$ \\
\hline $\begin{array}{l}\text { 2.2. Los contenidos se han tratado de } \\
\text { acuerdo al objetivo propuesto. }\end{array}$ & $1,42 \%$ & $2,13 \%$ & $21,99 \%$ & $36,17 \%$ & $38,30 \%$ \\
\hline $\begin{array}{l}\text { 2.3. La duración del taller ha sido } \\
\text { adecuada al objetivo propuesto. }\end{array}$ & $0,00 \%$ & $1,42 \%$ & $15,60 \%$ & $45,39 \%$ & $37,59 \%$ \\
\hline $\begin{array}{l}\text { 2.4. La duración del taller ha sido } \\
\text { adecuada a los contenidos } \\
\text { desarrollados. }\end{array}$ & & &
\end{tabular}

Fuente: Elaboración propia.

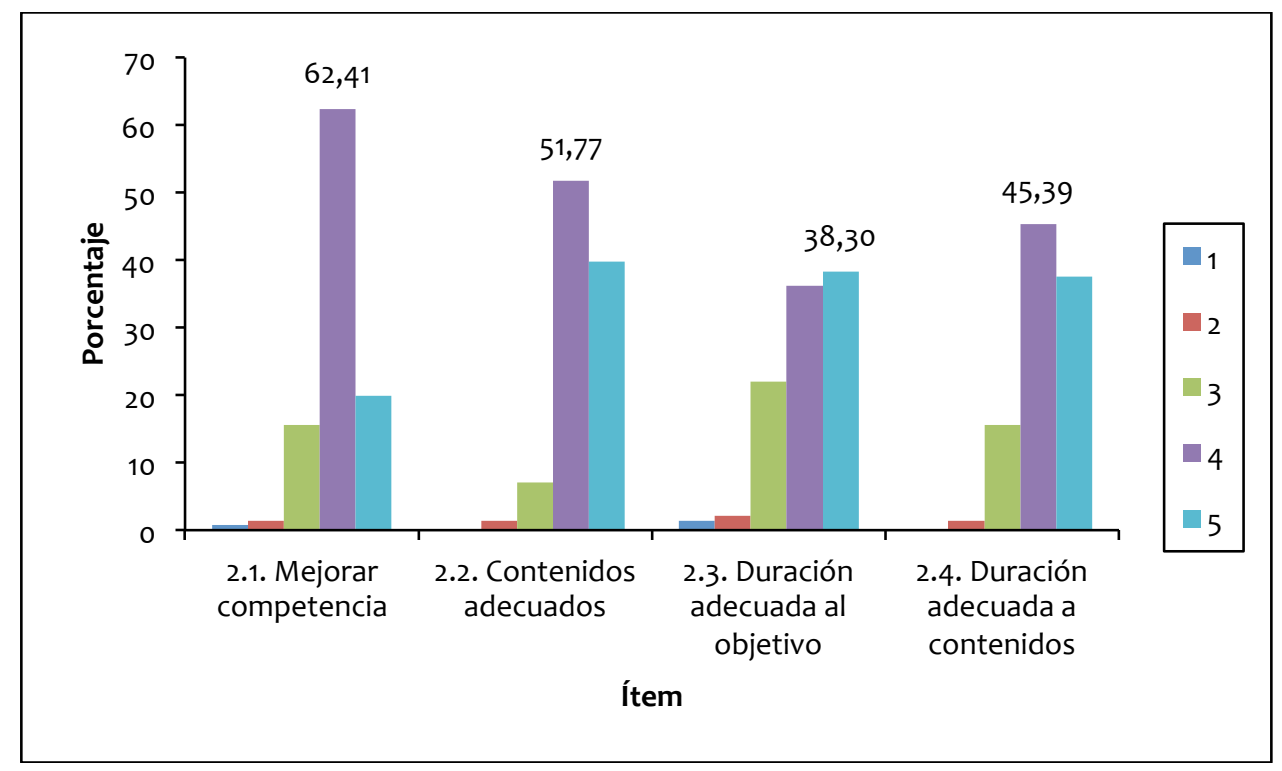

Gráfico 1. Sección Objetivos y contenidos. Fuente: Elaboración propia.

Como mencionábamos anteriormente, el análisis hecho de los datos extraídos de la encuesta nos ha permitido obtener información con respecto a la opinión de hombres y mujeres. En el gráfico 2 se pude ver que es mayor el porcentaje en el grupo de mujeres 
quienes están totalmente de acuerdo con los cuatro ítems correspondientes al apartado Objetivos y contenidos.

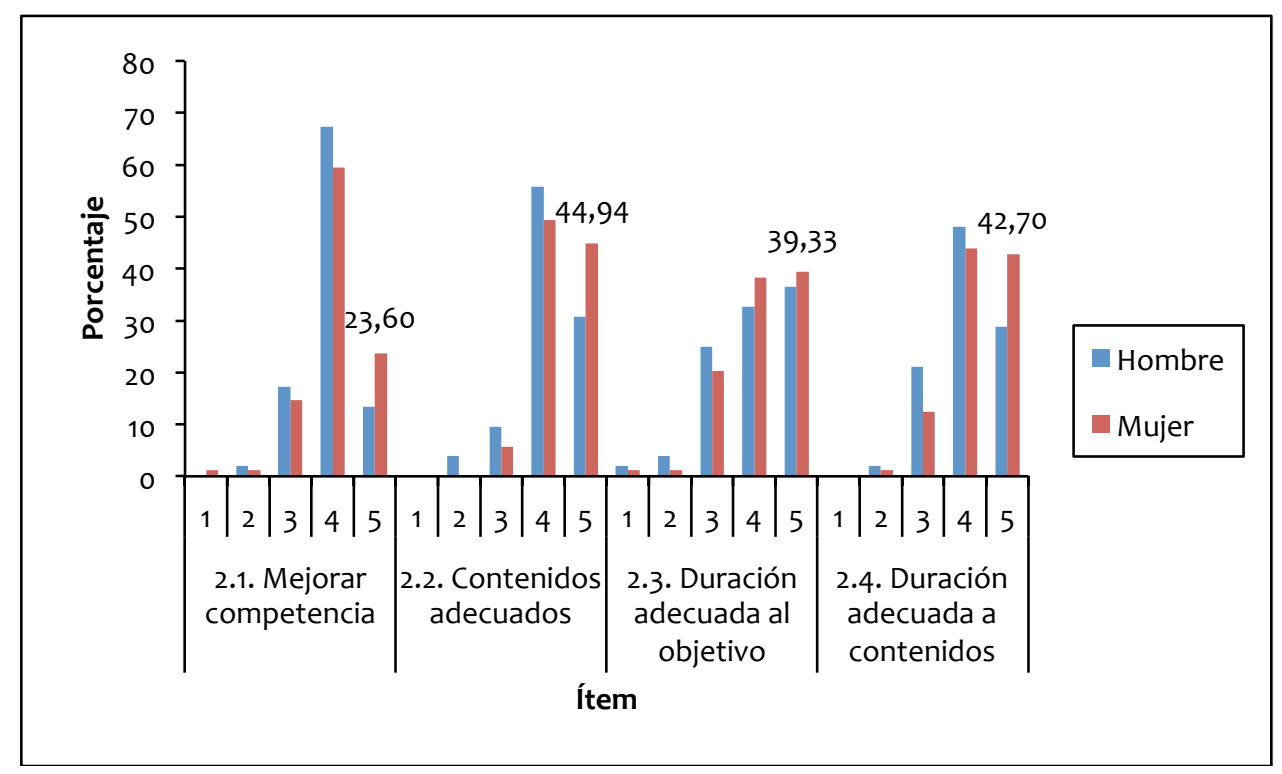

Gráfico 2. Sección Objetivos y contenidos según el sexo. Fuente: Elaboración propia.

\section{Metodología}

Un $46,81 \%$ está de acuerdo en que la metodología empelada se ha adecuado al objetivo propuesto. El 53,90\% están de acuerdo con la metodología seguida en relación a los contenidos trabajados. Están totalmente de acuerdo con el material didáctico utilizado un 43,97\%. En cuanto al último ítem de esta sección el 39,01\% están de acuerdo con que la metodología ha permitido una participación activa por parte de los asistentes. Véase tabla 3 y gráfico 3 .

Tabla 3.

Sección Metodología.

\begin{tabular}{|l|c|c|c|c|c|}
\hline \multirow{2}{*}{ ítem } & \multicolumn{5}{|c|}{ Nivel de acuerdo o desacuerdo } \\
\cline { 2 - 6 } & $\mathbf{1}$ & $\mathbf{2}$ & $\mathbf{3}$ & $\mathbf{4}$ & $\mathbf{5}$ \\
\hline $\begin{array}{l}\text { 3.1. La metodología desarrollada se ha } \\
\text { adecuado al objetivo propuesto. }\end{array}$ & $0,00 \%$ & $2,13 \%$ & $11,35 \%$ & $46,81 \%$ & $39,72 \%$ \\
\hline $\begin{array}{l}\text { 3.2. La metodología desarrollada se ha } \\
\text { adecuado a los contenidos expuestos. }\end{array}$ & $1,42 \%$ & $0,00 \%$ & $8,51 \%$ & $53,90 \%$ & $36,17 \%$ \\
\hline $\begin{array}{l}\text { 3.3. El material didáctico utilizado ha } \\
\text { sido adecuado para alcanzar el objetivo } \\
\text { del taller. }\end{array}$ & $0,71 \%$ & $2,84 \%$ & $9,93 \%$ & $42,55 \%$ & $43,97 \%$ \\
\hline $\begin{array}{l}\text { 3.4. La metodología ha permitido la } \\
\text { participación activa de los asistentes. }\end{array}$ & $2,13 \%$ & $7,80 \%$ & $12,77 \%$ & $39,01 \%$ & $38,30 \%$ \\
\hline
\end{tabular}

Fuente: Elaboración propia. 


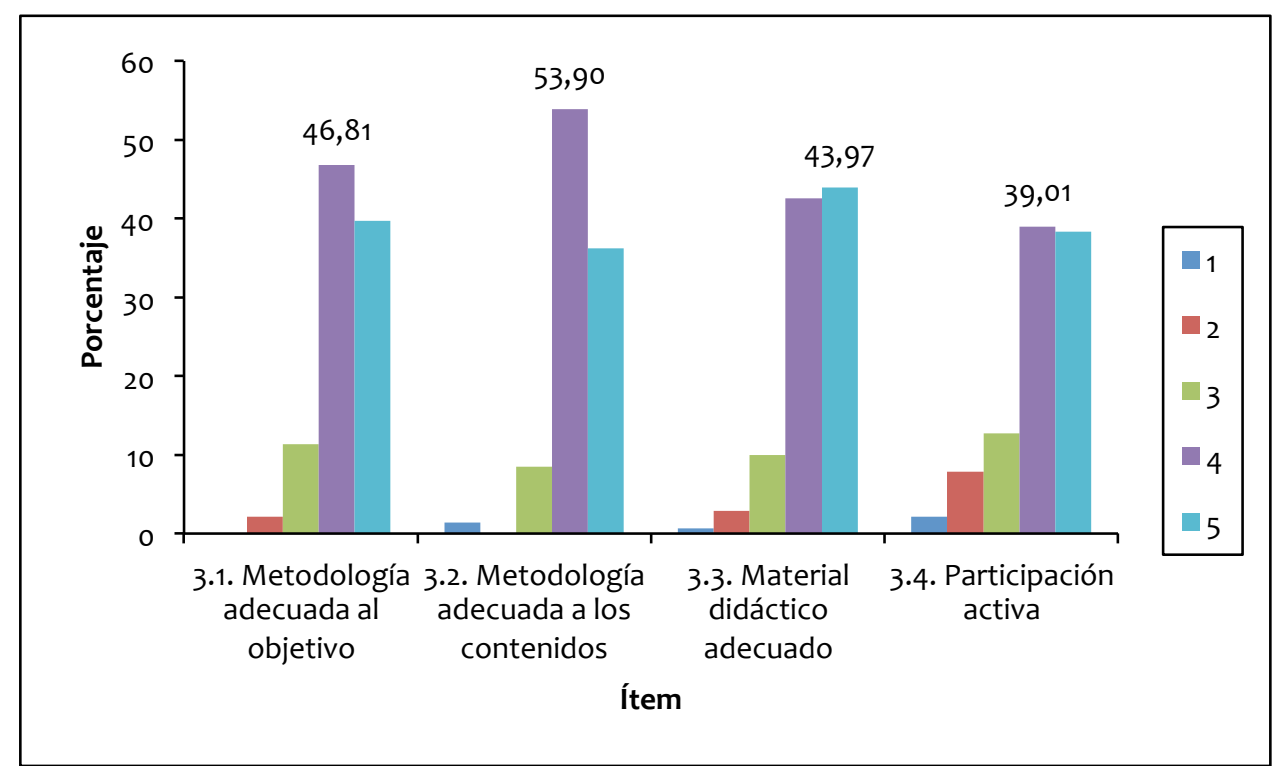

Gráfico 3. Sección Metodología.

Fuente: Elaboración propia.

Al igual que ocurría con el apartado Objetivos y contenidos, en el gráfico 4 vemos que es mayor el porcentaje en el grupo de mujeres quienes están totalmente de acuerdo con los cuatro ítems del apartado Metodología.

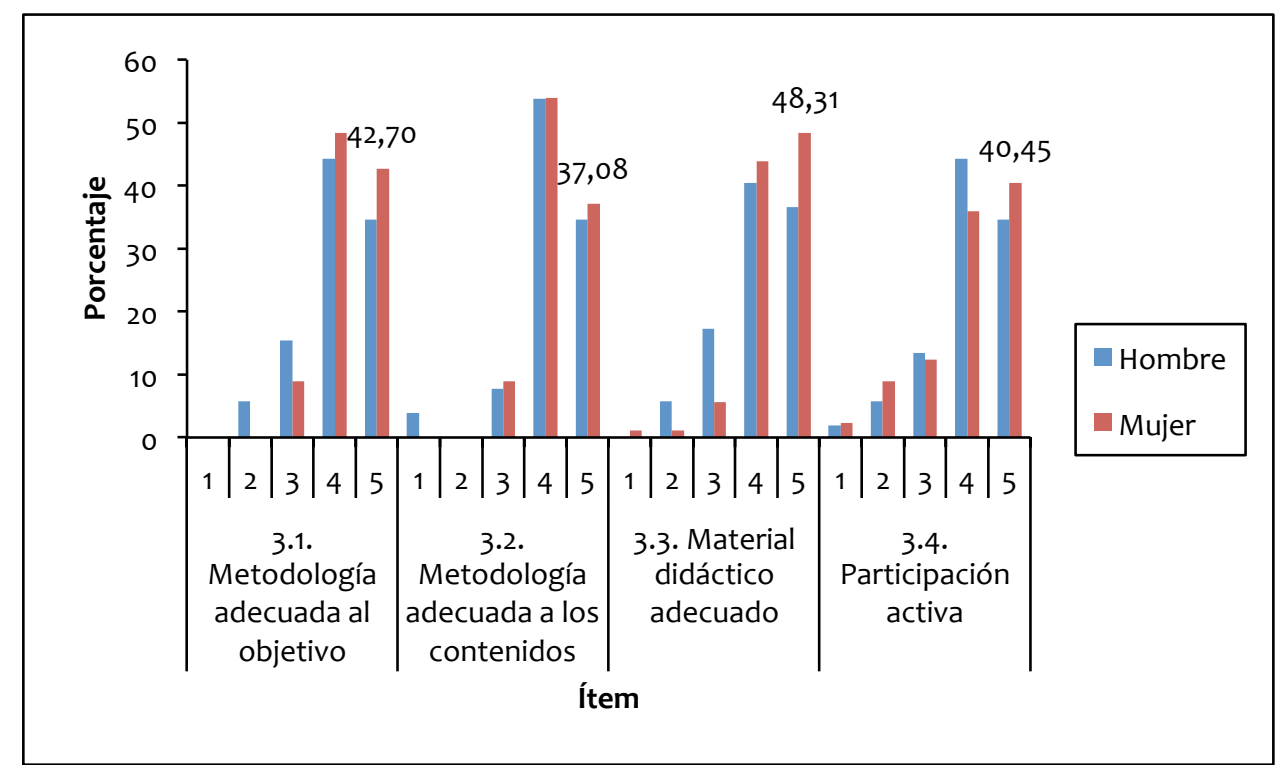

Gráfico 4. Metodología según el sexo.

Fuente: Elaboración propia 


\section{Utilidad, valoración global}

Como se aprecia en la tabla 4 y gráfico 5 , un $64,54 \%$ de los alumnos participantes consideran que el taller que han recibido es importante para su trabajo como docente.

Consideran, un $63,83 \%$, que el taller recibido les ha venido bien para su trabajo como alumnos. Incluso consideran, un $46,10 \%$ y $60,99 \%$ respectivamente, que recomendarían el taller a sus amigos y a sus compañeros de clase. El 43,97\% están de acuerdo en que el taller ha favorecido la exposición del trabajo que han tenido que presentar. El 59,57\% están totalmente de acuerdo que el taller merece una valoración global positiva.

Tabla 4.

Utilidad y valoración global.

\begin{tabular}{|c|c|c|c|c|c|}
\hline \multirow{2}{*}{ Ítem } & \multicolumn{5}{|c|}{ Nivel de acuerdo o desacuerdo } \\
\hline & 1 & 2 & 3 & 4 & 5 \\
\hline $\begin{array}{l}\text { 4.1. El taller recibido es importante para mi } \\
\text { trabajo como docente. }\end{array}$ & $0,71 \%$ & $0,71 \%$ & $4,26 \%$ & $29,79 \%$ & $64,54 \%$ \\
\hline $\begin{array}{l}\text { 4.2. Como alumno, me ha venido bien recibir } \\
\text { este taller. }\end{array}$ & $1,42 \%$ & $0,00 \%$ & $5,67 \%$ & $29,08 \%$ & $63,83 \%$ \\
\hline 4.3. Recomendaría este taller a mis amigos. & $1,42 \%$ & $2,84 \%$ & $12,77 \%$ & $36,88 \%$ & $46,10 \%$ \\
\hline $\begin{array}{l}\text { 4.4. Recomendaría este taller a mis } \\
\text { compañeros de clase. }\end{array}$ & $0,71 \%$ & $1,42 \%$ & $6,38 \%$ & $30,50 \%$ & $60,99 \%$ \\
\hline $\begin{array}{l}\text { 4.5. Creo que la exposición de mi trabajo ha } \\
\text { resultado mejor al haber recibido el taller de } \\
\text { hablar en público. }\end{array}$ & $1,42 \%$ & $2,84 \%$ & $12,77 \%$ & $43,97 \%$ & $39,01 \%$ \\
\hline $\begin{array}{l}\text { 4.6. El taller merece una valoración global } \\
\text { positiva. }\end{array}$ & $0,71 \%$ & $0,71 \%$ & $1,42 \%$ & $37,59 \%$ & $59,57 \%$ \\
\hline
\end{tabular}

Fuente: Elaboración propia.

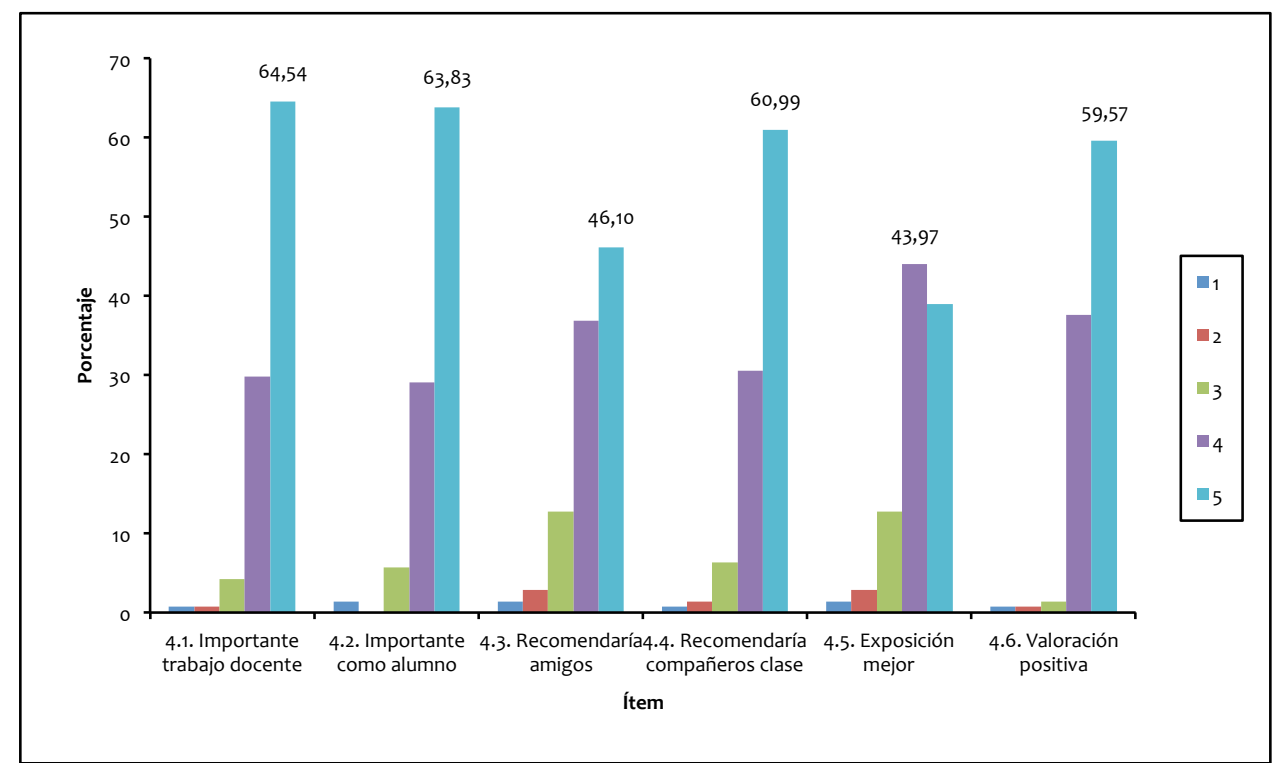

Gráfico 5. Utilidad y valoración global.

Fuente: Elaboración propia. 
Si apreciamos el gráfico 6, se vuelve a repetir el dato de que es mayor el porcentaje en el grupo de mujeres quienes están totalmente de acuerdo con los seis ítems del apartado Utilidad y valoración global.

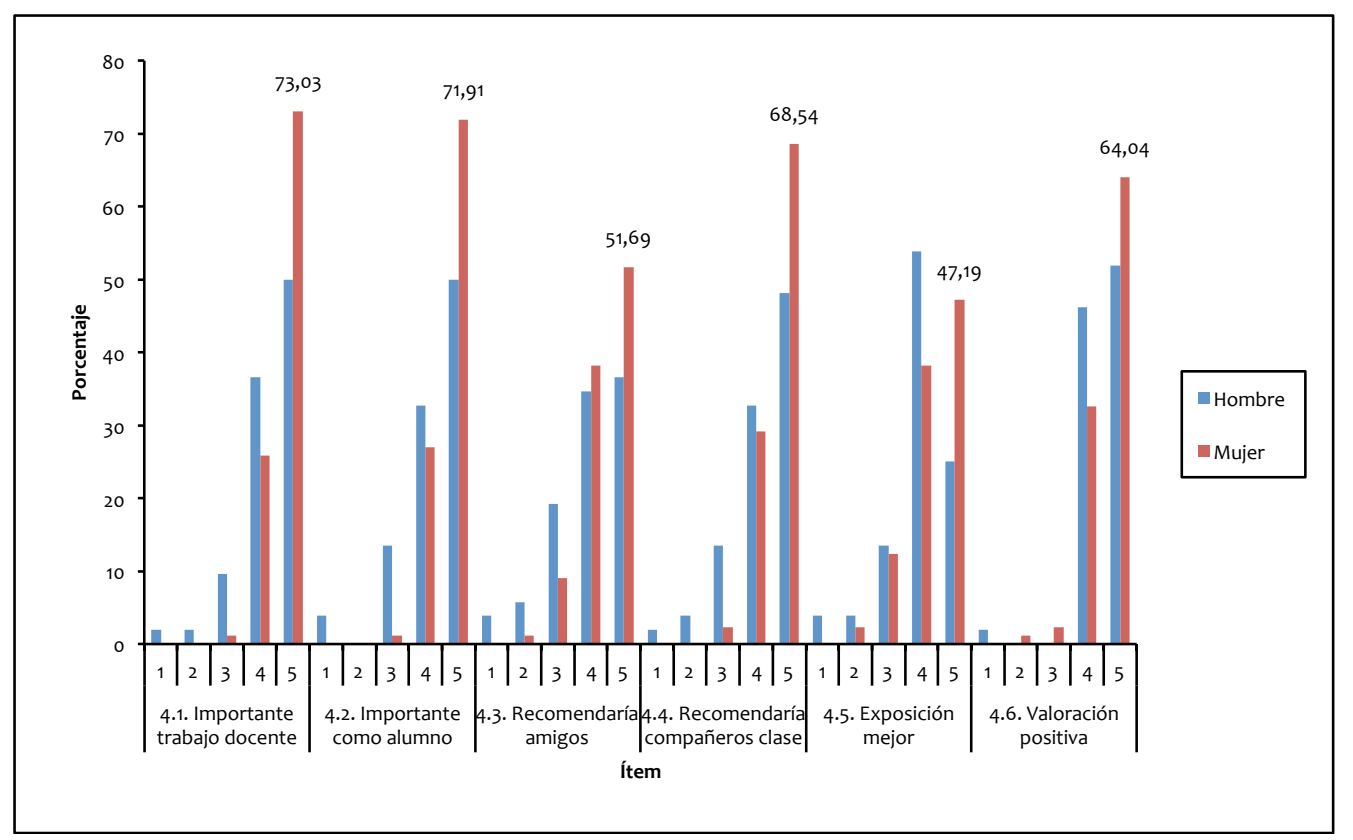

Gráfico 6. Utilidad y valoración global según el sexo.

Fuente: Elaboración propia.

\section{Conclusiones}

En esta experiencia formativa se pretendía dotar al alumnado universitario del Grado de Educación Primaria de la Facultad de Educación de la Universidad de Extremadura de los recursos necesarios que le permitan desarrollar la habilidad de hablar en público. Los resultados extraídos de la encuesta de satisfacción muestran la generalización del éxito del taller Hablar en público impartido a los estudiantes universitarios del Grado de Educación Primaria. Por ello, se debe considerar la posibilidad de ofertar este tipo de taller a los alumnos del Grado de Maestro, en general, con objeto de contribuir al desarrollo de la competencia transversal de hablar en público, y por tanto, a enriquecer sus potenciales como docentes $y$ al resto de personas que tengan que hacer exposiciones ante una audiencia y no dispongan de la habilidad de hablar en público, en particular. Además, como hemos indicado, los destinatarios de esta experiencia son alumnos de la Facultad de Educación, y por tanto, al finalizar sus estudios se convertirán en docentes. Esta es otra de las razones, dado el carácter de "leader" que todo docente debe ejercer, por la que es necesaria esta habilidad para trabajar con grupos de personas con objeto de introducir mejoras, transformaciones, y cambios en la práctica educativa. De hecho, si no se convence, no es posible iniciar ningún cambio.

No obstante, hay que señalar como puntos débiles del taller impartido que los asistentes al taller demandan una mayor duración del mismo para poder alcanzar la habilidad de hablar en público así como una participación más activa con respecto a la metodología desarrollada, ya que son menos de la mitad quiénes están totalmente de acuerdo con el tipo de participación dada a los alumnos y con la duración del taller $(38,30 \%$ y 38,30\% 
respectivamente). Por lo tanto, debemos contemplar para mejorar futuras ediciones del taller la posibilidad de ampliar la duración del mismo y proporcionar mayor participación a los asistentes en las actividades desarrolladas. También sería interesante indagar a través de entrevistas o grupos de discusión porqué los hombres parecen darle menos utilidad que las mujeres al taller recibido sobre hablar en público.

\section{Referencias}

Argyle, M. (1981). The contribution of social interaction research to social skills training. En J. D. Wine \& M. D. Smye (Eds.) Social competence (261-286). Nueva York: Guilford.

Bados, A. (1992). Análisis de componentes de un tratamiento cognitivo-somático-conductual del miedo a hablar en público. Barcelona: Publicaciones de la Universidad de Barcelona. (Tesis doctoral microfichada original de 1986).

Bados, A. (2005). Miedo a hablar en público. Universidad de Barcelona. Recuperado de http://diposit.ub.edu/dspace/bitstream/2445/353/1/120.pdf

Bang-Rouhet, P. (2009). ¿Qué nos jugamos cuando hablamos en público? Barcelona: Editorial Alienta.

Castaño, E., Blanco, A. y Asensio, E. (2012). Competencias para la tutoría: experiencia de formación con profesores universitarios. REDU. Revista de Docencia Universitaria, Número monográfico dedicado a Competencias docentes en la Educación Superior, 10 (2), 193-210.

D'El Rey, G. J. F. y Pacini, C. A. (2005). Tratamento da fobia social por exposição ao vivo e reestruturação cognitiva. Revista de Psiquiatria Clínica, 32, 231-235.

Fernández, M. A., González, J. L., López, I. y Manso, M. E. (2010). Evaluación participativa en habilidades para comunicar en $3^{\circ}$ de Grado de Enfermería en el curso 2009/10, la escenificación como método docente y de evaluación. Revista de Docencia Universitaria, 8 (2), 73-93.

Harris, K. R. y Brown, R. D. (1982). Cognitive behavior modification and informed teacher treatment for shy children. Journal of Experimental Education, 50, 137-143.

Lane, T. W. y Borkovec, T. D. (1984). The influence of therapeutic expectancy/demand on self-efficacy ratings. Cognitive Therapy and Research, 8, 95-106.

Lang, P. J., Levin, D. N., Miller G. A. y Kozak, M. J. (1983). Fear behavior, fear imagery and the psychophysiology of emotion: The problem of affective response integration. Journal of Abnormal Psychology, 92, 276-306.

Likert, R. (1932). A Technique for the Measurement of Attitudes. Archives of Psychology, 140, $1-55$.

Marina, J. A. (2006). Anatomía del miedo. Barcelona: Anagrama.

Marshall, W. L. (1981). Behavioral treatment of phobic and obsessive-compulsive disorders. En L. Michelson, M. Hersen \& S. M. Turner (Eds.). Future perspective in behavior therapy (161-186). Nueva York: Plenum.

Olivares, J. y García-López, L. J. (2002). Resultados a largo plazo de un tratamiento en grupo para el miedo a hablar en público. Psicothema, 14 (2), 405-409.

Puchol, L. (2008). Hablar en público: nuevas técnicas y recursos para influir a una audiencia en cualquier circunstancia. Ediciones Díaz de Santos. 
Stein, M. B., Walker, J. R. y Forde, D. R. (1994). Setting diagnostic thresholds for social phobia: Considerations from a community survey of social anxiety. American Journal of Psychiatry, 151, 408-412.

Wallace, I. Wallechinsky, D. y Wallace, A. (1977). The Book of Lists. New York: Bantam Books. 


\section{ENCUESTA DE SATISFACCIÓN Taller: Hablar en público}

ANEXO

Este cuestionario forma parte del Proyecto Desarrollo de competencias transversales en el aula universitaria. Hablar en público (código: B_2014_05), financiado en convocatoria pública competitiva por el Servicio de Orientación y Formación Docente de la Universidad de Extremadura dentro de la Convocatoria de Acciones para la Consolidación del Espacio Europeo de Educación Superior en la UEx (20132014). Está destinado a recoger información sobre las sesiones recibidas en el Taller: Hablar en público. Su opinión es importante. Por favor, responda con la máxima sinceridad.

Garantizamos la confidencialidad de los datos recogidos y agradecemos el interés y el tiempo disponible (cerca de cinco minutos) para responder a este cuestionario.

Fecha:

\section{Información personal}

Centro:

Titulación:

Especialidad:

Curso:

Grupo:

Edad:

Sexo: $\quad \mathrm{H}$

$\mathrm{M}$

Marca con una cruz en la casilla correspondiente, siendo $1=$ Totalmente en desacuerdo, $2=E n$ desacuerdo, $3=\mathrm{Ni}$ de acuerdo ni en desacuerdo, 4=De acuerdo y 5=Totalmente de acuerdo.

\section{Objetivos y contenidos}

2.1. En las sesiones realizadas se ha alcanzado el objetivo previsto: Mejorar la competencia transversal de hablar en público.

2.2. Los contenidos se han tratado de acuerdo al objetivo propuesto.

2.3. La duración del taller ha sido adecuada al objetivo propuesto.

2.4. La duración del taller ha sido adecuada a los contenidos desarrollados.

\section{Metodología}

3.1. La metodología desarrollada se ha adecuado al objetivo propuesto.

3.2. La metodología desarrollada se ha adecuado a los contenidos expuestos.

3.3. El material didáctico utilizado ha sido adecuado para alcanzar el objetivo del taller.

3.4. La metodología ha permitido la participación activa de los asistentes.

4. Utilidad y valoración global

4.1. El taller recibido es importante para mi trabajo como docente.

4.2. Como alumno, me ha venido bien recibir este taller.

4.3. Recomendaría este taller a mis amigos.

4.4. Recomendaría este taller a mis compañeros de clase.

4.5. Creo que la exposición de mi trabajo ha resultado mejor al haber recibido el taller de hablar en público.

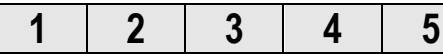

